\title{
HIV/AIDS among children in Ratodero, Pakistan amidst the COVID-19 pandemic: Challenges, efforts, and recommendations
}

A R T I C L E I N F O

\section{Keywords}

HIV

AIDS

Pakistan

COVID-19

\begin{abstract}
A B S T R A C T
In Pakistan, HIV has increased at an alarming rate in recent decades, and the death toll has risen. It is a public health challenge amidst the COVID-19 pandemic that is not being given due importance. The present article looks at the challenges and efforts being made to deal with HIV among children in Ratodero, Pakistan, and addresses measures that can be taken to control the disease in the child population epidemiologically.
\end{abstract}

\section{Introduction}

According to the World Health Organization (WHO), about 38 million people around the world are currently living with HIV/AIDS as of 2020, among whom 1.7 million are children under the age of 15 . Around 36.3 million people have also unfortunately succumbed to the disease till date. Even though the total number of AIDS-related deaths had decreased from 900,000 in 2015 to 680,000 in 2020 , but 1.5 million new infections were still recorded in 2020, averaging about 4000 new infections per day. About 150,000 new HIV infections were recorded in children less than 15 years in just a year 2020 alone. The majority of child and adult deaths due to HIV/AIDS have occurred in the regions of Africa, Asia, and the Pacific. ${ }^{1,2}$

In recent years, the HIV pandemic has had a tremendous effect on children. Around $10 \%$ of new HIV infections in the world are seen in children. The number of children infected with HIV has increased significantly since the first cases of AIDS were identified in 1982, mostly in developing countries. In 2010, an estimated 3.4 million children under the age of 15 were living with HIV globally, with approximately 240,000 new infections (58\% lower than in 2002) and an estimated 190,000 children dying from AIDS. ${ }^{3}$

In Pakistan, the burden of HIV has increased at an alarming rate in recent decades, and the death toll has risen. According to the National Health Survey report, Pakistan has an estimated 150,000 HIV patients, with 75,000 in Punjab, 15,000 in Khyber Pakhtunkhwa, and 5,000 in Baluchistan. By August 2018, Sindh had an estimated 60,000 HIVpositive patients. ${ }^{4}$

Unlike many other infectious diseases, HIV has a low prevalence in Pakistan, especially among children. ${ }^{5}$ However, in the first epidemiological study of data from 930 HIV-positive people diagnosed in Ratodero, a small town in Pakistan's Sindh province, between April 24 and July 15 , 2019, nearly $80 \%$ were children under the age of 5 . HIV infections in children were linked to receiving multiple injections i.e. same syringes were repeatedly used on one or more children from healthcare providers as part of treatment. ${ }^{6}$ This lead to an increase in HIV transmission increased due to this medical error which was mainly because of poorly trained staff. As of December 22, 2019, 1221 new HIV cases had been identified, 970 of which were in children, ${ }^{7}$ with the possible increase in number due to the increased rate of screening. According to the Sindh AIDS Control Programme (SACP), 42,533 people were screened by November 30,2020 , and 1,438 were found to be HIV positive and registered at the Ratodero ART Centre. Among them, there were 1,076 children in total, with 666 boys and 410 girls. The SACP has announced that 34 children and four adults have died due to the Ratodero outbreak. $^{8}$

\section{Challenges}

The average monthly household income in Pakistan is roughly $\$ 260$, but most people in Ratodero sustain on much less. ${ }^{9}$ In 2006, the cost of generic medicines for an HIV patient in Pakistan ranged from \$300 to $\$ 500$ ( $£ 174$ to $£ 291$; $€ 254$ to $424 €$ ), ${ }^{10}$ which would have increased if inflation had been factored in overtime. Most Ratodero inhabitants find it difficult to live comfortably whilst caring for an HIV-positive child as it adds to the financial strain of a local Ratodero family.

With the number of new HIV cases on the rise, there are significant barriers to access and adherence to antiretroviral therapy (ART). Even though the National AIDS Control Programme (NACP), with the help of the Global Fund, has developed 45 HIV treatment centers across the country's four provinces, ${ }^{11}$ people living with HIV often have to travel long distances to avail necessary treatment and pay out-of-pocket to cover for travel costs. ART centers are seldom managed by experts due to a shortage of infectious disease specialists. Physicians and other primary care professionals, in particular, lack expertise and training in the management of HIV-positive children. ${ }^{11}$

The possibility of ARTs running out of stock is also an added concern. ARTs are not produced in Pakistan and must be imported through a support mechanism set up by the Global Fund to Fight AIDS, Tuberculosis, and Malaria in Pakistan. Pediatric formulations of ART are susceptible to stockouts, as volumes of the supply required may not be as high as that of adult ART, therefore, leading to inadequacies in the planning of alternate supply chains in cases of shortages. ${ }^{12}$ HIV drug resistance is a serious global health problem that can have an effect on HIV treatment outcomes in low and middle-income countries with 
fractured healthcare systems, limited access to care, shortages of second-line ART regimens, and sub-optimal viral surveillance. ${ }^{13}$ HIV drug resistance can undermine the UNAIDS Fast Track goal of ending AIDS by $20300^{14-16}$

The COVID-19 pandemic has had an adverse impact on global healthcare and prevention delivery, and Pakistan is no exception. Pakistan had recorded 1,004,694 confirmed COVID-19 cases and 23,016 deaths as of July 25, 2021. ${ }^{17}$ People living with HIV with advanced disease as in those who are not on medication or not virally suppressed may be at higher risk of COVID-19 infection. ${ }^{18,19}$ Data on people afflicted with HIV and COVID-19 co-infection in Pakistan and deaths due to the same are currently not available.

During the implementation of a community engagement project in Ratodero's HIV-affected community, where over 1100 HIV positive children and adults are registered for ART, it was discovered that child mortality has increased in the last six months, and most children have not been tested for COVID-19 on personal communication with outreach workers of UNICEF and Sindh AIDS Control Programme who supported the project in Ratodero. It is unclear if COVID-19 has played a role in these negative outcomes, either directly through viral burden or indirectly through decreased access to treatment. ${ }^{20}$

\section{Efforts}

The Sindh AIDS Control Program (SACP) and the provincial Department of Health (DOH) have led the response to the incident. UN partners, the Pakistan Field Epidemiology \& Laboratory Training Program (FELTP), Aga Khan University, and other partners have all contributed to the response. Shaikh Zaid Children's Hospital in Lahore opened a new HIV/AIDS ART Treatment Center for children. Laboratories, blood banks, and clinics that were not approved have been shut down which was a step in the right direction to curb the outbreak. ${ }^{21}$

Throughout the first half of 2019, a mission led by the Federal Ministry of Health (MOH) and WHO, with help from other UN partners and academia, was carried out. The goals were to classify HIV transmission sources and chains, map high-risk areas, and identify gaps in HIV detection, care, and treatment. ${ }^{21}$ As a result of these efforts, the source of the outbreak was detected.

Officials mapped many families' back to a single doctor, thanks to the aforementioned actions and inquiries. Authorities arrested the doctor in late April 2019, accusing him of spreading the virus to hundreds of patients using contaminated syringes. The incident highlights how just a few syringes and one doctor can potentiate such disastrous consequences for patients as well as the community at large. Since needles can easily transmit viruses like HIV and hepatitis $\mathrm{C}$ between patients, reusing syringes in medical facilities is strictly prohibited. Syringes are however often still reused in Pakistan, even though it is illegal. ${ }^{22}$

\section{Recommendations}

Some patients must drive up to 7 hours to receive care for HIV at The Aga Khan Hospital, which is the only reputable medical facility in the region. With the support of UN agencies, the World Health Organization, and the Centers for Disease Control and Prevention, effective primary health care units should be built in the area to relieve the burden on families whose lives are already in shambles. ${ }^{23}$

Apart from supplying the facility only during pre-determined short periods of time, emergency services should also be made available 24 hours a day, seven days a week, because deaths may also be a result of the unavailability of routine emergency services. The healthcare system must be more cooperative, and the administration must be held responsible for any insufficient assistance that may be provided. ${ }^{24}$

Doctors should be evaluated on a regular basis to ensure their competency and alignment with current medical education and standards. Before issuing a license to practice, the physicians' mental and physical wellbeing should be evaluated as well to prevent the spread of further new cases of HIV. Testing kits and lab facilities are invaluable assets in the forefront for fighting diseases like HIV, because the disease cannot be effectively contained and tackled until they're found. According to reports, 3000 medical kits were distributed, which is insufficient for a district with more than 0.3 million people. These instruments and equipment, which include adequate sterile disposable syringes for the future, must also be borne in mind.Proper disposal of the used syringes should be done to prevent any future outbreak..$^{25,26}$

Though human life cannot be valued and compared with those of monetary benefits, a small financial contribution can make a big difference in helping a struggling family. According to a report, the average per capita income is less than 879 PKR per month, which is below the national poverty line. A family can also be helped with remuneration for the expenditure incurred as a result of medical services for HIV-infected children. The government can also help cover basic needs, such as food, housing, and healthcare for families who are currently below the poverty line. An extra monthly stipend may also be provided to families afflicted with HIV who are not under the poverty line but requiring some financial aid. ${ }^{27}$

Other recommendations include, (a) Increase in the number of HIV treatment centers, (b) Operation of all HIV treatment centers under the guidance of an infectious disease specialist, who can if necessary be virtual thanks to advancements in telemedicine during COVID-19, (c) Enhance staff training on the National Guidelines for ART and also assist health care providers in selecting suitable ART regimens, (d) Expand genotyping for HIV drug resistance (molecular surveillance), (e) Improve client counseling on ART, including community-based HIV treatment for people living with HIV and, (f) Advocate and ensure professionalism in HIV treatment centers at all levels to protect the privacy and human rights of people living with HIV and their families, (g) The children in the region should also be taught about sexually transmitted diseases transmission and regular screening should be done to prevent a future outbreak.

\section{Conclusion}

Since the prevalence of SARS-CoV-2 coinfection with HIV is unknown, governmental organizations such as the National AIDS Control Program (NACP), Community-Based Organizations (CBOs), and international agencies such as UNAIDS need to investigate the prevalence of coinfection with SARS-CoV-2 and HIV at the earliest to help prevent further adverse outcomes. People living with HIV should also be contacted to determine any disruptions in continuing their ART during the COVID-19 pandemic, as a result of economic adversities as well as actions taken by the government such as movement restrictions. Given that most HIV-positive individuals live on the outskirts of central cities and regions, it may be worthwhile to investigate the degree to which COVID-19-related preventive measures such as social distancing, mask use, and hand hygiene are being realistically adopted. While dealing with the COVID-19 pandemic, we must not overlook the biological and social vulnerabilities of people living with HIV in these trying times, especially in the context of widespread stigma and prejudice associated with the disease, as well as limited healthcare services. This goal can be achieved with timely analysis alongside quick and structured evidencebased action.

\section{Funding}

Not applicable.

\section{Data availability statement}

Not applicable. 


\section{Authors' contributions}

Anmol Mohan, Muhammad Taha Arshad Shaikh, and Um-ul-Wara developed the concept for this letter and wrote the original draft. Ana Carla dos Santos Costa, Sudhan Rackimuthu, and Priyanka Mohan Lal edited the second and final draft. Shoaib Ahmed, and Mohammad Yasir Essar made the critical comments and revisions. All authors revised and approved the final draft.

\section{Declaration of competing interest}

No conflict of interest declared.

\section{Acknowledgments}

None.

\section{References}

1. HIV/AIDS. World Health Organisation; 2020.

2. FactSheet- World Aids Day 2020. UNAIDS; 2020. https://www.unaids.org/sites/de fault/files/media_asset/UNAIDS_FactSheet_en.pdf.

3. Rabiu AT, Mohan A, Çavdaroğlu S, et al. Dengue and COVID-19: a double burden to Brazil. J Med Virol. 2021. https://doi.org/10.1002/jmv.26955 [Internet], (jmv.26955). Available from:.

4. Hussain A, Hussain S, Muhammad Ali S, Ali E, Mehmood A, Ali F. HIV/AIDS- a growing epidemic IN Pakistan. J Evol Med Dent Sci. 2018;7(8):1057-1062.

5. Pakistan country factsheet. UNAIDS. https://www.unaids.org/en/regionscountries /countries/pakistan; 2019.

6. Mir F, Mahmood F, Siddiqui AR, et al. HIV infection predominantly affecting children in Sindh, Pakistan, 2019: a cross-sectional study of an outbreak. Lancet Infect Dis. 2020;20(3):362-370.

7. Altaf A, Iqbal S, Shah SA. A third major human immunodeficiency viruses (HIV) outbreak in Larkana, Pakistan: caused by unsafe injection practices. J Pakistan Med Assoc. 2019;69(8):1068-1069.

8. Jajja S. The forgotten children of Ratodero. Dawn [Internet], [cited 2021 May 26]; Available from: https://www.dawn.com/news/1593308; 2020 Dec 1.

9. Ouyang H, Caron S. The city losing its children to H.i.v. The New York times [Internet], [cited 2021 May 26]; Available from: https://www.nytimes.com/2021/ 03/31/magazine/pakistan-hiv.html; 2021 Mar 31.

10. Yusufzai A. Pakistan starts HIV treatment programme. BMJ. 2006;332(7532):10.

11. Treatments - national AIDS control programme [Internet], Gov.pk. [cited 2021 May 26]. Available from: https://www.nacp.gov.pk/whatwedo/treatment.html.

12. Bravo MP, Peratikos MB, Muicha AS, et al. Monitoring pharmacy and test kit stocks in rural Mozambique: U.s. president's Emergency Plan for AIDS Relief surveillance to help prevent ministry of health shortages. AIDS Res Hum Retrovir. 2020;36(5): $415-426$.

13. World Health Organization. Meeting Report on Assessment of World Health Organization HIV Drug Resistance Early Warning Indicators: Report of the Early Advisory Indicator Panel Meeting, 11-12 August 2011. Geneva, Switzerland. Genève, Switzerland: World Health Organization; 2012.

14. HIV Drug Resistance. Avert; 2019. https://www.avert.org/professionals/hiv-progr amming/treatment/drug-resistance.

15. Steven YH, Jean BN, Karen K, Silvia B, Vincent CM, Michael RJ. The global status of HIV drug resistance: clinical and public-health approaches for detection, treatment and prevention. Infect Disord - Drug Targets. 2011;11(2):124-133.

16. Bennett DE, Jordan MR, Bertagnolio S, et al. HIV drug resistance early warning indicators in cohorts of individuals starting antiretroviral therapy between 2004 and
2009: world Health Organization global report from 50 countries. Clin Infect Dis. 2012;54(suppl_4):S280-S289.

17. Pakistan COVID: 939,931 cases and 21,633 deaths - worldometer [Internet], Worldometers.info. [cited 2021 Jun 12]. Available from: https://www.worldometer s.info/coronavirus/country/pakistan/rdination. https://covid.gov.pk/ [Internet]. Gov.pk. [cited 2021 May 26]. Available from:.

18. Coronavirus (COVID-19) and HIV. Avert; 2021. https://www.avert.org/coronavir us/covid19-HIV.

19. Hu Y, Ma J, Huang H, Vermund SH. Coinfection with HIV and SARS-CoV-2 in Wuhan, China: a 12-person case series. J Acquir Immune Defic Syndr. 2020;85(1):1-5.

20. WHO | HIV cases-Pakistan [cited 2021 May 26]; Available from: https://www.who. int/csr/don/03-july-2019-hiv-cases-pakistan/en/; 2019.

21. Jpma - journal of Pakistan medical association [Internet], Org.pk. [cited $2021 \mathrm{Jul}$ 27]. Available from: https://www.jpma.org.pk/article-details/10520.

22. Hussain S, Grady S. Hundreds of Children in Pakistan Have Been Infected with HIV. Used Needles Could Be to Blame. The washington Post; 2019.

23. The city losing its children to HIV [Internet], Akdn.org. [cited 2021 May 26]. Available from: https://www.akdn.org/media/city-losing-its-children-hiv.

24. HIV positive child dies in Rato Dero [Internet], Com.pk. 2021 [cited 2021 May 26]. Available from: https://tribune.com.pk/story/2282966/hiv-positive-child-dies-inrato-dero.

25. HIV horror in Pakistan's Sindh province as 157 test positive. Arab news [Internet], [cited 2021 May 26]; Available from https://www.arabnews.com/node/1492861/ pakistan; 2019 May 5.

26. District and tehsil level population summary with region breakup population NO OF HH district/tehsil region. https://www.pbs.gov.pk/sites/default/files/bwpsr/sindh/ LARKANA_SUMMARY.pdf.

27. Khan Sarmad, Ali Saadi Fazal. Socio-economic survey of ratodero Taluka district Larkana, Sindh. Rural support programmes network (RSPN). http://www.rspn. org/wp-content/uploads/2014/06/Socieconomic-Baseline-Survey-Ratodero-Taluk a-in-Sindh-2008.pdf; 2008.

Anmol Mohan*, Muhammad Taha Arshad Shaikh, Um-Ul Wara Karachi Medical \& Dental College, Karachi, Pakistan

Sudhan Rackimuthu

Father Muller Medical College, Mangalore, Karnataka, India E-mail address: sudhan.racki@gmail.com.

Ana Carla dos Santos Costa Federal University of Bahia - Salvador, Bahia, Brazil E-mail address: anacsc@ufba.br.

Priyanka Mohan Lal Ziauddin University, Karachi, Pakistan E-mail address: priyankamohan444@gmail.com.

Shoaib Ahmad Punjab Medical College, Faisalabad, Pakistan E-mail address: shoaibahmad442@gmail.com.

Mohammad Yasir Essar Medical Research Center, Kateb University, Kabul, Afghanistan E-mail address: m.yasir.essar@kateb.edu.af.

" Corresponding author.

E-mail addresses: anmolmohanvan@gmail.com (A. Mohan), tahaarshadshaikh@gmail.com (M.T.A.Shaikh), umulwara28@gmail. com (U.-U. Wara). 